\title{
73rd Annual Meeting of the American Academy of Neurology (AAN 2021): 17-22 April 2021
}

\author{
Sue Pochon ${ }^{1}$ \\ Published online: 28 May 2021 \\ (c) The Author(s), under exclusive licence to Springer Nature Switzerland AG 2021
}

For the second year running, as a result of the COVID-19 pandemic, the Annual Meeting of the American Academy of Neurology (AAN) was held virtually. The meeting was attended by over 13,000 people from nearly 115 countries, with the vast majority attending live.

The 6-day meeting offered an abundance of education and scientific programmes, as well as a first-rate series of plenary sessions. There were over 2000 abstracts and a similar number of posters presented during the course of the meeting and available through the virtual conference platform. In addition, there were over 100 exhibitors, and a host of networking and entertainment offerings to recreate the full conference experience for attendees.

This meeting report will provide a high-level overview of some of the most interesting clinical trials presented during the AAN plenary sessions.

\section{CENTAUR Trial in Amyotrophic Lateral Sclerosis}

Dr Sabrina Paganoni, Physician Investigator at the Healey Center for ALS at Massachusetts General Hospital (Boston, MA, USA), reported on the Phase 2 CENTAUR trial (NCT03127514) and discussed novel features of the trial's design, on behalf of the CENTAUR Study Group.

Clinical trials for amyotrophic lateral sclerosis (ALS) use a combination of survival and function endpoints to determine disease progression and response to therapy. Large and lengthy trials are typically needed, and there are serious associated issues, one being that declining ALS patients are kept on placebo for 12-18 months when many of these patients will not survive long enough to try the active drug.

Sue Pochon

sue.pochon@springer.com

1 Adis, Auckland, New Zealand
Function endpoints can also suffer from missing data as patients are lost to follow-up or die.

The CENTAUR trial, which was conducted at 25 sites in the USA, took a new, patient-centred approach aiming to minimise time spent on placebo, while still being able to gather meaningful data.

AMX0035 is a fixed-dose co-formulation of sodium phenylbutyrate (PB) and taurursodiol (TURSO) that has been shown to attenuate neuronal death and other pathology in cell cultures. The drug is formulated as a powder to be dissolved in water and administered orally or by feeding tube. It was taken once daily for the first 3 weeks in the CENTAUR trial and could be increased to twice daily if tolerated.

Currently, there are two drugs approved for ALS by the US Food and Drug Administration (FDA), riluzole and edaravone, and patients could remain on these standard-of-care drugs throughout the CENTAUR study, in keeping with the patient focus of the trial.

Initially, trial participants were randomised $2: 1$ to AMX0035 $(n=89)$ or placebo $(n=48)$ for a 6 -month period to measure safety, tolerability and impact on function outcomes. All participants from the double-blind portion of the trial were then invited to enrol into a long-term, openlabel follow-up study; $92 \%$ of completers from the first trial enrolled in the follow-up study and received AMX0035.

AMX0035 met its primary efficacy endpoint in the double-blind trial, with a significant difference between drug and placebo in ALSFRS-R total score at 24 weeks, indicating a slowing of functional decline [1]. Rates of adverse events (AEs) were similar between the two groups. The adjusted risks of death, tracheostomy and hospitalisation were not different between the groups at 24 weeks; however, Dr Paganoni explained that very few deaths or tracheostomies were recorded in this period.

In order to better assess long-term survival, the study group used a number of methods, including a participant locating service to track down outcomes data for every original participant in the CENTAUR trial $(n=137)$, including 
those who withdrew, were lost to follow-up or did not enrol in the open-label extension.

Pre-specified survival analysis compared time to death (all-cause mortality) in those originally randomised to AMX0035 and those originally randomised to placebo and found that those initiated on the study drug had a significant $44 \%$ lower risk of death than those who started the trial on placebo [2]. In the long term, risks of other significant events such as tracheostomy, permanent assisted ventilation or risk of first hospitalisation, were also significantly lower in AMX0035 subjects compared with the placebo subjects.

Given that most patients switched to AMX0035 at 6 months, these pre-specified survival analyses may underestimate the true survival benefit.

Dr Paganoni also presented a new exploratory analysis that compared observed survival with predicted survival based on the ENCALS Survival Prediction Model [3]. Results suggested an even greater survival benefit than the pre-specified survival analysis. Additional analyses showed lower rates of hospitalisation and dependence on mechanical ventilation in patients who received the active drug [4].

Amylyx have recently announced their intention to enrol patients into a Phase III trial of AMX0035 for ALS conducted in Europe and the USA. They also intend to file a New Drug Submission for AMY0035 with Health Canada in the first half of 2021.

\section{ESCAPE NA-1 Trial for Acute Ischaemic Stroke}

Dr Michael Tymianski, from NoNO Inc. in Toronto (ON, Canada), reported on ESCAPE NA-1 (NCT02930018), a multicentre, randomised, placebo-controlled Phase 3 trial of nerinetide in stroke patients who were selected for endovascular thrombectomy [5].

Participants $(n=1105)$ were stratified accordingly to device used (i.e., stent or aspiration device) and, more importantly, whether they had $(n=659)$ or had not $(n=446)$ received prior alteplase.

The primary outcome of the trial was the Modified Rankin Score (mRS), categorised as good function $9 \mathrm{mRS}$ $0-2$ ) or bad function (mRS $>3$ ), at 90 days.

Results showed that for the entire study population there was only a small, non-statistically significant, benefit in favour of nerinetide. However, pharmacokinetic data from a subset of participants in the ESCAPE-NA-1 trial showed that prior alteplase significantly reduced nerinetide plasma concentrations by up to $60 \%$, resulting in no effect in the stratum of patients who had received alteplase.

In contrast, in the participants who had not received alteplase, nerinetide significantly improved functional outcomes, reduced stroke mortality by $40 \%$ and reduced infarction volume by $22 \%$.

There will now be a follow-up trial called ESCAPENEXT (Nerinetide EXcept for Thrombolysis; NCT04462536), which will enrol endovascular thrombectomy candidates who have not received thrombolytics.

\section{ADVANCE Trial for the Prevention of Migraine}

Dr Jessica Ailani, Director of the MEDSTAR Georgetown Headache Center (Washington, DC, USA), reported on the Phase 3, multicentre, randomised, placebo-controlled, parallel group ADVANCE trial (NCT03777059) to assess the efficacy, safety and tolerability of atogepant for the prevention of migraine [6]. Atogepant is an experimental, oral small molecule CGRP receptor antagonist.

Participants $(n=910)$ in ADVANCE had to have between 4 and 14 migraine days per month in order to enrol and this was confirmed in a 4-week baseline screening period. The screening period was followed by a 12-week double-blind treatment period, where subjects were randomised 1:1:1:1 to placebo or one of three different once-daily doses of atogepant $(10 \mathrm{mg}, 20 \mathrm{mg}$ and $30 \mathrm{mg}$ ). This was then followed by a 4-week follow-up period.

The primary endpoint of the trial was change from baseline in the mean monthly migraine days across the 12-week treatment period. Secondary measures included headache days, acute medication use days, $50 \%$ responder rate, quality-of-life measures, performance of daily activities and physical impairment.

Across the 12-week treatment period, all atogepant groups met the primary efficacy endpoint and the highest two doses met all six secondary measures. The lowest dose group met four of the six secondary measures, missing on performance of daily activities and physical impairment measures.

There was a clinically meaningful and statistically significant reduction in mean monthly migraine days for all atogepant doses, with an overall therapeutic gain of 1.72 migraine days versus placebo for the highest dose and 1.38 migraine days for the 20-mg dose. Atogepant also resulted in $56-61 \%$ of participants achieving $\geq 50 \%$ reduction in monthly migraine days compared with $29 \%$ of placebo subjects.

The effect was rapid, with all three atogepant doses showing significant reduction from baseline in mean monthly migraine days from Week 1 of treatment. In fact, atogepant was considered effective in 1 day.

Atogepant was also considered safe and well tolerated. There was one single serious case of optic neuritis considered related to the study drug, but not confirmed clinically 
by investigational studies. No hepatic safety issues were identified.

Allergan have filed a New Drug Application for atogepant for the preventive treatment of migraine in adults with episodic migraine in the USA. A regulatory decision is expected in Q3 2021.

\section{Randomised Withdrawal Study for Idiopathic Hypersomnia}

Professor Yves Dauvilliers from University of Montpellier (Montpellier, France), reported on the Phase 3, placebocontrolled, double-blind, randomised withdrawal study (NCT03533114) of JZP-258, a lower-sodium oxybate (LXB) in adult participants with idiopathic hypersomnia (IH) [7].

Participants could be aged between 18 and 75 years of age, with IH according to ICSD-2 or ICSD-3 criteria and $\geq 7 \mathrm{~h}$ average nocturnal sleep time to exclude sleep deprivation. Subjects could either be treatment naïve or taking other medications for IH symptoms, and received a different treatment regimen with JZP-258 during the 4-week screening period depending on which of four other IH medications they were taking.

All participants then underwent a 10- to 12-week titration and optimisation period for efficacy and tolerability followed by a 2-week stable dose period. After this period, subjects were randomised 1:1 to continue taking JZP-258 or to take placebo for 2 weeks during the double-blind withdrawal period. Participants who completed this double-blind period were eligible for an open-label extension of 24 weeks.

The primary efficacy endpoint of the study was the change in Epworth Sleepiness Scale (ESS) score between the end of the stable dose period and the end of the doubleblind withdrawal period. Key secondary endpoints were the proportion of participants who worsened on Participant Global Impression of Change (PGIc) and change in Idiopathic Hypersomnia Severity Scale (IHSS) total score, over the same time period as the primary endpoint.

In total, 154 subjects were enrolled in the trial. Study participants who continued taking LXB saw an improvement in ESS score between the end of the stable dose period and the end of the double-blind withdrawal period, while subjects assigned to placebo had a worsening score. The difference between the groups was both highly significant and clinically relevant.

For secondary endpoints, significant worsening of the PGIc score was observed for patients on placebo compared with JZP-258. Likewise, participants on placebo reported mean worsening of IHSS score, while subjects on LXB reported maintenance or improvement of score, and the difference between the groups was highly statistically significant at the end of the double-blind withdrawal period.
Four participants reported serious events during the trial, including rhabdomyolysis, nephrolithiasis and syncope, but none were deemed to be related to the study drug. Twentyseven subjects also reported AEs leading to discontinuation.

JZP-258 has been launched by Jazz Pharmaceuticals for the treatment of cataplexy or excessive daytime sleepiness in patients aged $\geq 7$ years with narcolepsy in the USA. The drug is currently under rolling priority review for the treatment of IH in the USA. A decision is expected in the coming months.

\section{ADAPT Study for Generalised Myasthenia Gravis}

Dr James F. Howard, Professor at the University of North Carolina at Chapel Hill reported efficacy, safety and tolerability data from the Phase 3 ADAPT study (NCT03669588) of efgartigimod in patients with generalised myasthenia gravis (MG) [8].

In order to understand the mechanism underlying efgartigimod in MG, Dr Howard began his talk by explaining that the neonatal $\mathrm{Fc}$ receptor $(\mathrm{FcRn})$ is known to recycle $\mathrm{IgG}$, thus extending its half-life and serum concentration. Efgartigimod is a human IgG1 $\mathrm{Fc}$ fragment and a natural ligand of FcRn. Efgartigimod was designed with increased affinity to FcRn allowing it to outcompete endogenous FcRn.

The ADAPT study enrolled participants with MGFA class II, II or IV generalised MG, who were either acetylcholine receptor (AChR)-antibody positive or negative, had a Myasthenia Gravis Activities of Daily Living (MG-ADL) score of $\geq 5$ and were taking at least one stable MG treatment.

Trial subjects were randomised 1:1 to receive efgartigi$\bmod (n=84)$ or placebo $(n=83)$. Four doses were administered at weekly intervals and patients were observed for clinical effect. Patients who failed to respond to the first cycle of treatment or whose response subsequently declined could receive up to 3 cycles of treatment during the 26-week study duration. Treatment was individualised, with the time between cycles determined by the duration of clinically meaningful improvement. However, patients who needed rescue therapy were discontinued from the study treatment. The randomised double-blind phase was then followed by an open-label extension.

The primary endpoint of the trial was MG-ADL responders who were defined as having $\mathrm{a} \geq 2$-point improvement for at least 4 consecutive weeks in AChR-antibody positive patients in the first cycle (8 weeks).

Study results demonstrated a statistically significant clinical benefit. The primary endpoint was met by $67.7 \%$ of participants in the efgartigimod arm versus $29.7 \%$ in the placebo arm. Furthermore, a clear majority of those who 
were classed as MG-ADL responders had onset of effect within the first 2 weeks.

The secondary endpoint of the trial was Quantitative Myasthenia Gravis (QMG) score responders, who were defined as having a $\geq 3$-point improvement for at least 4 consecutive weeks in AChR-antibody positive patients in the first cycle ( 8 weeks).

Again, the endpoint was met, with $63.1 \%$ of treated subjects and $14.1 \%$ of placebo patients meeting responder criteria.

Dr Howard also showed that $>30 \%$ of efgartigimodtreated patients who did not respond in cycle one, were subsequently classed as MG ADL responders in cycle two. Considering both cycles one and two, 78.5\% of efgartigimod-treated patients were classed as MG ADL responders.

Adverse events were predominantly mild or moderate in severity and efgartigimod was generally well tolerated. Three subjects in each arm withdrew from the trial due to AEs.

Argenx have submitted a Biologics License Application for efgartigimod for the treatment of generalised MG in the USA. A regulatory decision is expected in December 2021.

\section{COMET Trial in Late-onset Pompe Disease}

Dr Hani Kushlaf, Associate Professor from the University of Cincinnati (OH, USA), reported on the COMET trial (NCT02782741), a Phase 3 multicentre, randomised, double-blind study comparing the efficacy and safety of intravenous avalglucosidase alfa with alglucosidase alfa in patients with late-onset Pompe disease [9].

Avalglucosidase alfa is a recombinant human $\alpha$-glucosidase enzyme replacement therapy, designed to reduce the glycogen accumulation caused by Pompe disease. Avalglucosidase alfa increases glycogen clearance and offers potential improvement on the clinical efficacy achieved with alglucosidase alfa due to its ability to enter cells more easily, thereby allowing for faster and more efficient processing of glycogen.

Enrolled participants across 20 countries had to be at least 3 years of age, with a diagnosis of Pompe disease, and naïve to alglucosidase alfa or any investigational therapy for Pompe disease. They were also required to perform the necessary FVC measurements and ambulate $40 \mathrm{~m}$.

The primary objective of the study was to determine the effect of avalglucosidase alfa on respiratory muscle function compared with standard of care alglucosidase alfa and the primary endpoint was measured by $\%$ predicted FVC in the upright position.

Secondary objectives were to determine the effect of avalglucosidase alfa treatment on functional endurance, inspiratory and expiratory muscle strength and pressures, lower extremity muscle strength, motor function and health-related quality of life. Safety was also assessed, along with pharmacokinetic, pharmacodynamics and pharmacogenetics.

In total, 100 participants were randomised 1:1 to avalglucosidase alfa $(n=51)$ or alglucosidase alfa $(n=49)$ and 95 participants entered the extended treatment period. Of the five participants who discontinued, four did so for AEs and one participant withdrew consent. All five subjects were in the alglucosidase alfa arm of the study.

Treatment with avalglucosidase alfa resulted in a $2.43 \%$ greater improvement in \% predicted FVC at Week 49 compared with alglucosidase alfa. This result was statistically significant for non-inferiority but non-significant for superiority versus alglucosidase alfa.

Clinically and statistically significant improvement was also seen in the main secondary endpoint, the 6-min walk test, with a notable increase of $30 \mathrm{~m}$ in the distance covered. Positive numerical trends were seen for the other secondary endpoints, which include muscle measures, functional endurance and health-related quality of life.

Avalglucosidase alfa appears to have a favourable safety and tolerability profile compared with alglucosidase alfa.

Dr Kushlaf concluded that these data offer clinical evidence of substantial improvement with avalglucosidase alfa over alglucosidase alfa in patients with late-onset Pompe disease.

Avalglucosidase alfa, which is being developed by Sanofi Genzyme, is currently under regulatory review in both the USA and EU for the treatment of patients with Pompe disease. A US FDA decision is expected in August 2021 and the EU decision is expected in the second half of 2021.

\section{Next Meeting}

The 74th Annual Meeting of the AAN is expected to be held in Seattle, WA, USA from 2nd to 8th April 2022.

\section{Declarations}

Funding No funding was received for this meeting report.

Conflicts of interest/Competing interest The author has no conflicts of interest to declare.

Ethics approval Not applicable.

Consent to participate Not applicable.

Consent for publication Not applicable.

Availability of data and material Not applicable.

Code availability Not applicable. 
Author contributions The author was the sole contributor to this meeting report and approved the final published article.

\section{References}

1. Paganoni S, Macklin EA, Hendrix S, et al. Trial of sodium phenylbutyrate-taurursodiol for amyotrophic lateral sclerosis. N Engl J Med. 2020;383:919-30.

2. Paganoni S, Hendrix S, Dickson SP, et al. Long-term survival of participants in the CENTAUR trial of sodium phenylbutyratetaurursodiol in amyotrophic lateral sclerosis. Muscle Nerve. 2021;63:31-9.

3. Paganoni S, Hendrix S, Dickson $S$, et al. Long-term survival of participants in the CENTAUR trial of AMX0035 for ALS [abstract]. 2021 Annual Meeting of the American Academy of Neurology (AAN). 17-22 April; Virtual. https://index.mirasmart. com/AAN2021/index.php. Accessed 5 May 2021.

4. Paganoni S, Macklin EA, Hendrix S, et al. Lower long-term risk of death or permanent ventilation and first hospitalization among participants with ALS receiving AMX0035 in the CENTAUR trial[abstract]. 2021 Annual Meeting of the American Academy of Neurology (AAN). 17-22 April; Virtual. https://index.miras mart.com/AAN2021/index.php. Accessed 5 May 2021.

5. Hill MD, Goyal M, Menon BK, et al. Efficacy and safety of nerinetide for the treatment of acute ischaemic stroke
(ESCAPE-NA1): a multicentre, double-blind, randomised controlled trial. Lancet. 2020;395(10227):878-87.

6. Ailani J, Lipton R, Goadsby PJ, et al. Atogepant Significantly Reduces Mean Monthly Migraine Days in the Phase 3 Trial (ADVANCE) for the Preventive Treatment of Migraine [abstract]. 2021 Annual Meeting of the American Academy of Neurology (AAN). 17-22 April; Virtual. https://index.mirasmart.com/ AAN2021/index.php. Accessed 5 May 2021.

7. Dauvilliers Y, Arnulf I, Foldvary-Schaefer N, at el. Efficacy and safety of lower-sodium oxybate in a phase 3, placebo-controlled, double-blind, randomized withdrawal study in adult participants with idiopathic hypersomnia [abstract]. 2021 Annual Meeting of the American Academy of Neurology (AAN). 17-22 April; Virtual. https://index.mirasmart.com/AAN2021/index.php. Accessed 5 May 2021.

8. Howard J, Bril V, Vu T, et al. Efficacy, safety, and tolerability of efgartigimod in patients with generalized myasthenia gravis: analysis of the phase 3 ADAPT Study [abstract]. 2021 Annual Meeting of the American Academy of Neurology (AAN). 17-22 April; Virtual. https://index.mirasmart.com/AAN2021/index.php. Accessed 5 May 2021.

9. Kushlaf H, Attarian S, Borges JL, et al. Efficacy and safety results of the avalglucosidase alfa phase 3 COMET trial in late-onset Pompe disease patients [abstract]. 2021 Annual Meeting of the American Academy of Neurology (AAN). 17-22 April; Virtual. https://index.mirasmart.com/AAN2021/index.php. Accessed 5 May 2021. 